\title{
ANOTHER NOTE ON PARACOMPACT SPACES
}

\author{
E. MICHAEL 1
}

1. Introduction. The purpose of this paper is to obtain some new characterizations of paracompact spaces, one of which implies that the image of a paracompact space, under a continuous and closed mapping, must be paracompact. This answers the author's Research Problem 29 in [4].

Call a collection $a$ of subsets of a topological space closure-preserving if, for every subcollection $B \subset Q$, the union of closures is the closure of the union (i.e. $\cup\{\bar{B} \mid B \in B\}=[\cup\{B \mid B \in B\}]^{-}$). Any locally finite $^{2}$ collection is certainly closure-preserving, but the converse is generally false even for discrete spaces. Nevertheless, it will be shown that, both in the definition of paracompactness and in the characterizations obtained by the author in [3], "locally finite" can be replaced by "closure-preserving." This implies the corollary mentioned above, since the image of a closure-preserving collection under a closed mapping is again closure-preserving.

In the statements of the theorems below, a covering (and a refinement) is a collection of sets which covers the space; its elements need not be open unless that is specifically assumed. ${ }^{3}$ Theorem 1 strengthens [3, Lemma 1], and Theorem 2 strengthens [3, Theorem 1].

THEOREM 1. The following properties of a regular topological space $X$ are equivalent:

(a) $X$ is paracompact. ${ }^{4}$

(b) Every open covering of $X$ has a closure-preserving open refinement.

(c) Every open covering of $X$ has a closure-preserving refinement.

Presented to the American Mathematical Society April 28, 1956 under the title Some new characterizations of paracompactness; received by the editors February 16, 1956 and, in revised form, April 2, 1956.

1 This paper was written while the author was a member of a National Science Foundation project at the University of Washington.

2 A collection $Q$ of subsets of $X$ is locally finite if every $x \in X$ has a neighborhood intersecting only finitely many elements of $\mathcal{Q}$.

3 This might be the place to point out that all the coverings (and refinements) appearing in the diagram on p. 835 of [3] should have been labeled open.

- A Hausdorff space is paracompact if every open covering has a locally finite open refinement. 
(d) Every open covering of $X$ has a closure-preserving closed refinement.

COROllaRy 1. The image of a paracompact space, under a continuous, closed mapping, must be paracompact.

TheOREM 2. A regular topological space is paracompact if and only if every open covering has a $\sigma$-closure-preserving open refinement. ${ }^{5}$

Theorem 2 raises the question of whether one can also replace " $\sigma$-locally finite" by " $\sigma$-closure-preserving" in the Nagata-Smirnov $[5 ; 6]$ characterization of metrizability for regular spaces (i.e. there exists a $\sigma$-locally finite base for the open sets). The answer is "no," as is shown by the subset of the Stone-Cech compactification of the space $N$ of integers consisting of $N$ and one point $x \notin N$.

Concerning Corollary 1 , it should be remarked that the analogous result for normal spaces was proved in a three-line proof by G. T. Whyburn [7, Theorem 9], and that similar easy proofs can be given for many other familiar types of spaces: collectionwise normal, perfectly normal, normal and countably paracompact. ${ }^{6}$ Of course the proof of Corollary 1 is equally trivial, but only after one has the characterization of paracompactness in Theorem 1 (c) or (d).

The only difficult proof in this paper is that (d) implies (a) in Theorem 1; this will be shown in $\$ 2$. The remainder of Theorem 1 $((\mathrm{a}) \rightarrow(\mathrm{b}) \rightarrow(\mathrm{c}) \rightarrow(\mathrm{d}))$ is trivial, and Corollary 1 and Theorem 2 are easy consequences of Theorem 1 ; the proofs will be given in $\$ \S 3,4$, and 5 .

2. Proof that $(d) \rightarrow(a)$ in Theorem 1 . Throughout this section, we shall assume that the $T_{1}$-space ${ }^{7} X$ satisfies (d) of Theorem 1. Our aim is to prove that $X$ must be paracompact, by showing every open cover $\mathcal{U}$ of $X$ has a $\sigma$-locally finite (in fact $\sigma$-discrete) ${ }^{8}$ open refinement $W$; this is sufficient by [3, Theorem 1$]$. We begin with two lemmas.

${ }^{5}$ Following recent trends in terminology, we call a collection $\mathcal{U}$ of subsets $\sigma$ closure-preserving if $V=\bigcup_{i-1}^{\infty} V_{i}$, with each $\mho_{i}$ closure-preserving. It should be observed that, as the proof shows, the theorem's requirement that the elements of the refinement $\mathcal{V}$ be open can be weakened to require only that each $\mathcal{V}_{i}$ have an open union.

- Using Dowker's characterization [1, Theorem 2]: Every countable open covering $\left\{U_{i}\right\}_{i=1}^{\infty}$ has a closed refinement $\left\{A_{i}\right\}_{i=1}^{\infty}$ with $A_{i} \subset U_{i}$ for all $i$.

${ }^{7}$ For this part of Theorem 1 , one need not assume that $X$ is regular.

$8 W$ is discrete if every $x \in X$ has a neighborhood intersecting at most one $W \in W$; it is $\sigma$-discrete (resp. $\sigma$-locally finite) if $W=\bigcup_{i=1}^{\infty} W_{i}$, with each $W_{i}$ discrete (resp. locally finite). 
LemMA 1. If $\left\{U_{\alpha}\right\}_{\alpha \in \mathbf{A}}$ is an indexed open covering of $X$, then there exists an indexed closure-preserving closed covering $\left\{C_{\alpha}\right\}_{\alpha \in \mathrm{A}}$ of $X$ such that $C_{\alpha} \subset U_{\alpha}$ for all $\alpha$.

Proof. By assumption, $\left\{U_{\alpha}\right\}_{\alpha \in \mathrm{A}}$ has a closure-preserving closed refinement $B$. For each $B \in B$, pick an $\alpha(B)$ such that $B \subset U_{\alpha(B)}$. For every $\alpha$, let

$$
C_{\alpha}=\bigcup\{B \in B \mid \alpha(B)=\alpha\} .
$$

Then $C_{\alpha} \subset U_{\alpha}$ for all $\alpha$, and $\left\{C_{\alpha}\right\}_{\alpha \in \mathbf{A}}$ is a closure-preserving closed covering of $X$ because $B$ is. This completes the proof.

Lemma 2. $X$ is normal.

Proof. Let $E_{1}, E_{2}$ be disjoint, closed subsets of $X$. Then $\left\{X-E_{1}, X-E_{2}\right\}$ is an open covering of $X$, and hence there exists a closed covering $\left\{C_{1}, C_{2}\right\}$ of $X$ such that $C_{i} \subset\left(X-E_{i}\right)$ for $i=1,2$. But then the open sets $X-C_{1}$ and $X-C_{2}$ separate $E_{1}$ and $E_{2}$, and the proof is complete.

After these preliminaries, let $\left\{U_{\alpha}\right\}_{\alpha \in \mathbf{A}}$ be an open covering of $X$ which, for convenience, has been indexed by a well-ordered index set $\mathrm{A}$. We must show that this covering has a $\sigma$-locally finite open refinement.

Our first step is to construct, for each positive integer $i$, a family $\left\{C_{\alpha, i}\right\}_{\alpha \in \mathrm{A}}$ of subsets of $X$ satisfying the following conditions for all $i$ :

(1) $\left\{C_{\alpha, i}\right\}_{\alpha \in \mathbf{A}}$ is a closure-preserving closed covering of $X$, and $C_{\alpha, i} \subset U_{\alpha}$ for all $\alpha$.

(2) $C_{\alpha, i+1} \cap C_{\beta, i}=\varnothing$ for all $\alpha>\beta$.

The construction is simple. A covering $\left\{C_{\alpha, 1}\right\}_{\alpha \in \mathbf{A}}$, satisfying (1) for $i=1$, can be found by Lemma 1 . Suppose that coverings $\left\{C_{\alpha, i}\right\}_{\alpha \in A}$ have been picked to satisfy our conditions for $i=1, \cdots, n$, and let us construct $\left\{C_{\alpha, n+1}\right\}_{\alpha \in \mathbf{A}}$. Let

$$
U_{\alpha, n+1}=U_{\alpha}-\left(\bigcup_{\beta<\alpha} C_{\beta, n}\right)
$$

for all $\alpha \in \mathrm{A}$. The sets $U_{\alpha, n+1}$ are open because $\left\{C_{\alpha, n}\right\}_{\alpha \in \mathrm{A}}$ is closure preserving, and they cover $X$ because, if $x \in X$, then $x \in U_{\alpha, n+1}$ for the first $\alpha$ for which $x \in U_{\alpha}$. We now use Lemma 1 to pick a closurepreserving, closed covering $\left\{C_{\alpha, n+1}\right\}_{\alpha \in \mathrm{A}}$ of $X$ such that $C_{\alpha, n+1} \subset U_{\alpha, n+1}$ for all $\alpha$. Condition (1) is obviously satisfied for $i=n+1$, and (2) holds for $i=n$ by the definition of the sets $U_{\alpha, n+1}$ and the fact that $C_{\alpha, n+1} \subset U_{\alpha, n+1}$ for all $\alpha$.

For each $\alpha$ and each $i$, we now let 


$$
V_{\alpha, i}=X-\left(\bigcup_{\beta \neq \alpha} C_{\beta, i}\right)
$$

Let us show that

(3) $\left\{V_{\alpha, i} \mid \alpha \in A, i=1,2, \cdots\right\}$ is an open covering of $X$, and $V_{\alpha, i} \subset U_{\alpha}$ for all $\alpha$ and $i$.

(4) $V_{\alpha, i} \cap V_{\beta, i}=\varnothing$ whenever $\alpha \neq \beta$.

To see this, note that each $V_{\alpha, i}$ is open because $\left\{C_{\beta, i}\right\}_{\beta \in \mathrm{A}}$ is closurepreserving. Since $\left\{C_{\alpha, i}\right\}_{\alpha \in \mathrm{A}}$ is a covering of $X$, we have

$$
V_{\alpha, i} \subset C_{\alpha, i} \subset U_{\alpha}
$$

for all $\alpha$ and $i$, and this, together with the definition of $V_{\alpha, i}$, implies (4). All that remains to be proved is that the sets $V_{\alpha, i}$ cover $X$. To show this, pick an $x \in X$, and let us find a $V_{\alpha, i}$ containing it. Using the well-orderedness of the index set $A$, we let

$$
\alpha_{i}=\min \left\{\alpha \in A \mid x \in C_{\alpha, i}\right\}, \quad i=1,2, \cdots,
$$

and then pick a positive integer $k$ such that

$$
\alpha_{k}=\min \left\{\alpha_{i} \mid i=1,2, \cdots\right\} \text {. }
$$

But now

$$
x \in V_{\boldsymbol{\alpha}_{k}, k+1},
$$

because $x \notin C_{\alpha, k+1}$ for $\alpha<\alpha_{k}$ by the definition of $\alpha_{k}$, and $x \notin C_{\alpha, k+1}$ for $\alpha>\alpha_{k}$ by (2) (with $i=k$ and $\beta=\alpha_{k}$ ) and the fact that $x \in C_{\alpha_{k}, k}$ by definition of $\alpha_{k}$.

To complete the proof, we apply Lemma 1 once more to obtain a closure-preserving closed covering $\left\{D_{\alpha, i} \mid \alpha \in \mathrm{A}, i=1,2, \cdots\right\}$ of $X$ such that

$$
D_{\alpha, i} \subset V_{\alpha, i}
$$

for all $\alpha$ and $i$. For each $i$, we can now apply a lemma of C. $H$. Dowker [2] ${ }^{9}$ (remembering that $X$ is normal by Lemma 2 ) to obtain a discrete ${ }^{8}$ family $\left\{W_{\alpha, i}\right\}_{\alpha \in \mathbf{A}}$ of open sets such that

$$
D_{\alpha, i} \subset W_{\alpha, i} \subset V_{\alpha, i}
$$

for all $\alpha$. The collection $\left\{W_{\alpha, i} \mid \alpha \in A, i=1,2, \cdots\right\}$ is now the required $\sigma$-discrete open refinement of $\left\{U_{\alpha}\right\}_{\alpha \in A}$, and the proof is complete.

We conclude the section with the statement and proof of the lemma of Dowker which was used above.

- While this lemma was essentially proved in the middle of p. 308 of [2], it was not stated explicitly, and for completeness we therefore state and prove it below. 
LemMa 3 (Dowker). If $\left\{V_{\lambda}\right\}_{\lambda \in \Lambda}$ is a disjoint collection of open subsets of a normal space $X$, if $D_{\lambda} \subset V_{\lambda}$ for each $\lambda$, and if $\bigcup_{\lambda \in \Lambda} D_{\lambda}$ is closed, then there exists a discrete ${ }^{8}$ family $\left\{W_{\lambda}\right\}_{\lambda \in \Delta}$ of open subsets of $X$ such that

$$
D_{\lambda} \subset W_{\lambda} \subset V_{\lambda}
$$

for all $\lambda$.

Proof. Let $S=\{x \in X \mid$ some neighborhood of $x$ intersects at most one $\left.V_{\lambda}\right\}$. Then $S$ is open, and contains the closed set $U_{\lambda \in \Lambda} D_{\lambda}$. By normality, there exists an open $R \subset X$ such that

$$
\left(\bigcup_{\lambda \in \Delta} D_{\lambda}\right) \subset R \subset \bar{R} \subset S
$$

and now the sets

$$
W_{\lambda}=V_{\lambda} \cap R
$$

satisfy all our requirements.

3. Proof that $(a) \rightarrow(b) \rightarrow(c) \rightarrow(d)$ in Theorem 1. That $(a) \rightarrow(b)$ follows from the introductory remark that every locally finite collection is closure-preserving. That (b) $\rightarrow$ (c) is obvious. To show that $(c) \rightarrow(d)$, let $v$ be an open covering of $X$. Since $X$ is regular (this is the only place in the proof of Theorem 1 where this assumption is used), there exists an open covering $W$ of $X$ such that $\{\bar{W} \mid W \in W\}$ is a refinement of $v$. By assumption (c), there now exists a closure-preserving refinement of $R$ of $w$. But then $\{\bar{R} \mid R \in R\}$ is a closure-preserving closed refinement of $\mathcal{V}$, and this proves (d).

4. Proof of Corollary 1. Let $X$ be paracompact, and let $f$ be a continuous, closed map from $X$ onto a topological space $Y$. We must show that $Y$ is paracompact. Since $Y$ is certainly $T_{1}$, it suffices, by Theorem 1 and footnote 7 , to show that every open covering $V$ of $Y$ has a closure-preserving closed refinement. Let $W=\left\{f^{-1}(V) \mid V \in V\right\}$; then $W$ is an open covering of $X$, and hence, by paracompactness, has a closure-preserving closed refinement $a$. But then

$$
\{f(A) \mid A \in a\}
$$

is the required closure-preserving closed refinement of $V$, and the proof is complete.

5. Proof of Theorem 2. This proof is quite similar to the proof of [3, Theorem 1]. To prove the nontrivial part of the theorem, let us assume that $X$ is a regular space, every open covering of which has a 
$\sigma$-closure-preserving open refinement; we must show that $X$ is paracompact. By Theorem 1, we need only show that every open covering $W$ of $X$ has a closure-preserving refinement $B$. We begin with two elementary lemmas about closure-preserving collections, whose proof can be left to the reader.

LEMma 4. If $\mathcal{F}$ is a closure-preserving collection of closed subsets of a topological space $X$, and if $B \subset X$ is closed, then $\{F \cap B \mid F \in \mathcal{F}\}$ is also closure-preserving.

Leмma 5. If $\left\{C_{\alpha}\right\}_{\alpha \in \mathbf{A}}$ is a locally finite collection of subsets of a topological space $X$, and if, for every $\alpha, \bigotimes_{\alpha}$ is a closure-preserving (with respect to $X)$ collection of subsets of $C_{\alpha}$, then $\bigcup\left\{\Theta_{\alpha} \mid \alpha \in \mathrm{A}\right\}$ is also closurepreserving.

To prove the theorem, let $R$ be an open covering of $X$. By the regularity of $X$, there exists an open covering $S$ of $X$ such that $\{\bar{S} \mid S \in \mathcal{S}\}$ is a refinement of $R$. By assumption, $\delta$ has an open refinement $V=U_{i=1}^{\infty} V_{i}$, where each $V_{i}$ is closure-preserving. Let

$$
V_{i}=U\left\{V \mid V \in v_{i}\right\} \quad i=1,2, \cdots
$$

and let

$$
\begin{aligned}
& C_{1}=X, \\
& C_{i}=X-\bigcup_{j=1}^{i-1} V_{j} \quad i=2,3, \cdots .
\end{aligned}
$$

Observe that each $C_{i}$ is closed, and that $\left\{C_{i}\right\}_{i=1}^{\infty}$ is locally finite. Now finally let

$$
\begin{aligned}
B_{i} & =\left\{\bar{V} \cap C_{i} \mid V \in V_{i}\right\} \quad i=1,2, \cdots \\
B & =\bigcup_{i=1}^{\infty} B_{i} .
\end{aligned}
$$

Then certainly $B$ is a refinement of $R$. To see that it is closurepreserving, observe that each $B_{i}$ is closure-preserving by Lemma 4 , and hence so is $B$ by Lemma 5 and the fact that every element of $B_{i}$ is a subset of $C_{i}$. This completes the proof.

\section{BIBLIOGRAPHY}

1. C. H. Dowker, On countably paracompact spaces, Canadian J. of Math. vol. 3 (1951) pp. 219-224. 313.

2. - On a theorem of Hanner, Arkiv för Matematik vol. 2 (1952) pp. 307-

3. E. Michael, A note on paracompact spaces, Proc. Amer. Math. Soc. vol. 4 (1953) pp. 831-838. 
4. - - Paracompact spaces 29, Bull. Amer Math. Soc. Research Problem 61-6-29.

5. J. Nagata, On a necessary and sufficient condition of metrizability, J. Inst. Polytech., Osaka City Univ., Ser. A. Math. vol. 1 (1950) pp. 93-100.

6. Yu. Smirnov, A necessary and sufficient condition for metrizability of a topological space, Doklady Akad. Nauk. SSSR. N.S. vol. 77 (1951) pp. 197-200.

7. G. T. Whyburn, Open and closed mappings, Duke Math. J. vol. 17 (1950) pp. 69-74.

\section{UNIVERSITY OF WASHINGTON}

\section{COMPACT MAPPINGS}

EDWIN HALFAR

In the Memoir Open mappings on locally compact spaces, G. T. Whyburn shows the equivalence of his definition of compact mappings and that of E. A. Vainstein [1] under the assumption that the spaces involved are separable and metric. In the following, it is shown that the two are equivalent on more general spaces provided that the range space is suitably restricted. The remainder of the note is concerned with a result about the relation between closedness of a mapping and continuity and with an elaboration of a result concerning quasi-interior mappings.

In general, the spaces are $T_{1}$ unless otherwise stated. The mapping (i.e. continuous function) $f(X)=Y$ is closed if $F$ a closed subset of $X$ implies $f(F)$ is closed; $f$ is compact if $K$ a compact subset of $Y$ implies $f^{-1}(K)$ is a compact subset of $X ; f$ is quasi-interior if for any $y \in Y$ and any open set $U$ in $X$ which contains a compact component of $f^{-1}(y), y$ is interior to $f(U) . A$ is an inverse set if $A=f^{-1}(A)$. Also, $G_{0}$ will denote the union of all sets of the type $f^{-1}(y)$ which are subsets of $G$.

Theorem 1. If $f(X)=Y$ is a closed mapping and if for each $y \in Y$, $f^{-1}(y)$ is compact, then $f$ is compact.

Let $K$ be a compact subset of $Y$ and $g$ a covering of $f^{-1}(K)$. Since $f$ is closed, for each open subset $G$ of $X, G_{0}$ is an open inverse set, and $f\left(G_{0}\right)$ is an open subset of $Y$. For some $y \in K, f^{-1}(y)$ may fail to be a subset of a single element of $\mathcal{G}$, but since $f^{-1}(y)$ is compact, a finite subcollection $\mathcal{G}_{1}$ of $g$ will cover $f^{-1}(y)$. Let $G^{*}$ be the union of the elements of $g_{1}$. Then $G_{0}^{*}$ is a non-null open inverse set. Thus, the collec-

Received by the editors July 9, 1956 and, in revised form, December 5, 1956. 